ACUTE KIDNEY INJURY

\section{DNA origami scavenges ROS in the kidney}

Acute kidney injury (AKI) can be triggered by a variety of insults, including exposure to iodinated contrast media, which has a nephrotoxic effect. Patients who are imaging procedures are normally treated with antioxidants to prevent AKI, but the effectiveness of these interventions is uncertain. An alternative approach might come in the form of DNA origami nanostructures (DONs) that accumulate in the kidney, according to findings just published by Peng Huang, Chunhai Fan, Hao Yan, Weibo Cai and colleagues.

"The base-pairing principles of DNA enable the rational design of DNA structures on the nanometer and sizes," explains Yan, "but their biodistribution patterns in living systems have not yet been fully established." To investigate the behaviour of DONs in vivo, the researchers radiolabelled DONs that had rectangular, triangular or tubular shapes and used positron emission tomography (PET) imaging to track them in healthy animals. They found that these origami nanostructures accumulated in the administered contrast media for scale with programmable shapes

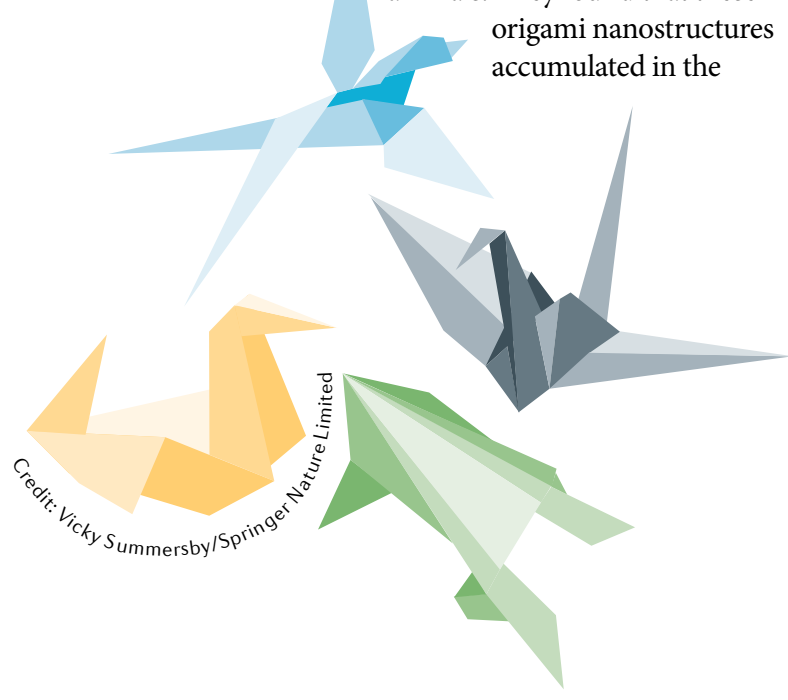

kidney and were completely cleared $24 \mathrm{~h}$ post-injection. By contrast, short single-stranded DNA (ssDNA) was rapidly cleared in the kidney whereas long ssDNA and partially folded DNA structures accumulated in the liver. "DONs with different frameworks could be rationally designed to specifically target different organs," suggests Fan.

Rectangular DONs (Rec-DONs) had the best renal uptake and confocal imaging of kidneys harvested at $10 \mathrm{~min}, 3 \mathrm{~h}$ and $12 \mathrm{~h}$ post-injection demonstrated that they first accumulate in the glomeruli and then in the renal tubules. Rec-DONs not only accumulated in the kidney of healthy mice as early as $5 \mathrm{~min}$ post-injection, but also in the kidneys of mice with rhabdomyolysis-induced AKI, in which they had a protective effect. In this mouse model, kidney injury is caused by reactive oxygen species (ROS) that are released from damaged muscle cells and excreted in the kidney, where they damage renal cells and eventually lead to AKI. "DNA is sensitive to ROS and many investigators have searched for the optimal antioxidants to protect DNA from ROS damage," notes Cai. "By contrast, in this model, we used extraneous DNA from DONs as an antioxidant to neutralize ROS."

The effects of Rec-DON injection in the kidney were compared with those of long ssDNA, as well as to high $(4.2 \mathrm{mg})$ and low $(10 \mu \mathrm{g})$ doses of the antioxidant $\mathrm{N}$-acetylcysteine (NAC), which is commonly used to treat contrast-induced AKI. Kidney function was assessed by quantitative renal PET imaging with ${ }^{68} \mathrm{Ga}$-ethylenediaminetetraacetic acid (EDTA), which is retained in the blood with minimal renal excretion in the context of severe kidney damage. In addition, kidney and blood samples were collected and analysed $24 \mathrm{~h}$ after AKI induction. Treatment with Rec-DONs or with high-dose NAC prevented weight loss and reduced the levels of creatinine and blood urea nitrogen in comparison to the other interventions; kidney tissue sections stained with haematoxylin and eosin also evidenced reduced kidney damage.

Reductants such as superoxide dismutase (SOD) can neutralize ROS and prevent cellular damage. The levels of SOD were reduced in animals treated with PBS, ssDNA or low-dose NAC, whereas treatment with Rec-DONs or high-dose NAC restored SOD levels to those of healthy animals.

Collectively, these data suggest that Rec-DONs are as effective as $\mathrm{NAC}$ at protecting the kidney from rhabdomyolysis-induced AKI. However, when the doses of NAC and Rec-DONs were adjusted to $10 \mu \mathrm{g}$ and $20 \mu \mathrm{g}$, respectively, so that they contained equal levels of reductant molecules, only Rec-DONs were protective. Higher doses of NAC ( $4.2 \mathrm{mg}$ ) were required to achieve the same effect.

Injection of DONs did not trigger the production of IL- 6 or tumour necrosis factor, suggesting low immunogenicity. There was also no evidence that DON administration had a negative effect on kidney or liver function, and tissue sections of the heart, liver, spleen, lung and kidneys showed no abnormalities at $24 \mathrm{~h}$, suggesting low toxicity.

"Recently published methods should enable cost reduction in the chemical synthesis of DNA so we envision that this new smart nanomaterial has great potential for translational application, not just as a drug carrier but as an effective therapy itself," notes Huang.

Monica Wang

ORIGINAL ARTICLE Jiang, D. et al. DNA origami nanostructures can exhibit preferential renal uptake and alleviate acute kidney injury. Nat. Biomed. Eng. 2,865-877 (2018) 